\title{
Optical properties of ferroelectric photonic structures
}

\author{
D. Bi ${ }^{1}$, V.S. Gorelik ${ }^{1,2}$ \\ ${ }^{1}$ Bauman Moscow State Technical University, 105005, Moscow, Russia \\ ${ }^{2}$ P.N. Lebedev Physical Institute of the Russian Academy of Sciences, 119991, Moscow, Russia \\ gorelik@sci.lebedev.ru
}

Ferroelectric materials have been an important issue in physical research since their discovery. Their optical properties have attracted the interest of many researchers. A ferroelectric photonic structure can be fabricated by filling a ferroelectric substance into a mesoporous photonic crystal, which can enhance the possibility of observing optical phenomena in the ferroelectric structure [1]. This paper presents the research results of the optical properties of ferroelectric photonic structures.

The initial samples studied in this work are opal matrices, constructed from highly packed amorphous silica nanoglobules. The opal matrix was formed by self-assembly [2] of silica nanospheres prepared by the Stober's method [3]. Ferroelectric substances $\left(\mathrm{NaNO}_{2}, \mathrm{KNO}_{3}\right.$, etc.) were filled into the cavity of the photonic crystal by placing mesoporous photonic crystals into the ferroelectric substance solution and evaporation with heating.

In the reflection spectra of photonic crystals, there are strong reflective areas of light, i.e., photonic stop band [4-6]. The positions of the stop band depend on the refractive index, the crystal lattice constant of the constituent material, etc. After introducing different ferroelectric substances into the cavity of the photonic crystal, the formed composite ferroelectric photonic structures have different dielectric constants. Therefore, for the photonic composite structures made from different ferroelectric materials and the same initial photonic crystal, the shifts in stop band positions are different. The shift in stop band positions of the composite photonic structure is also related to the amount of ferroelectric material filled.

Raman spectra of composite ferroelectric photonic structures and their corresponding ferroelectric materials were observed in this work. To reduce the influence of the fluorescence spectrum of opal matrix, the exciting laser of Raman scattering is chosen to be $785 \mathrm{~nm}$. When the incident light of the Raman spectrum was close to the stop band position of the composite photonic crystals, the density of the photonic states was greatly enhanced [7]. In addition, there was also Mie scattering in the opal matrix with large-sized particles, which also caused a sharp increase in the density of photonic states [8]. Thus, the Raman spectral intensity of the ferroelectric composite photonic crystal was increased. Such optical property of composite opal photonic crystals can be used to increase the probability of occurrence of other optical phenomena in them, such as second harmonic generation [9] and others [10, 11]. This plays an important role in expanding the application of ferroelectric materials.

Thus, it is very important to prepare new ferroelectric photonic composite structures and study their optical properties. It can help to find new applications of ferroelectric materials in optical or other aspects.

This study was supported by the RFBR (№ 18-02-00181) and the China Scholarship Council.

1. Y. Almohamed, R. Barille, A.I. Vodchits, et al., JETP Lett. 101, 365 (2015).

2. C.K. Liau, Y.K. Huang, Expert Systems with Applications 35, 887 (2008).

3. W. StoBer, A. Fink, E. Bohn, J. Colloid Interface Sci. 26, 62 (1968).

4. E. Yablonovitch, Phys. Rev. Lett. 58, 2059 (1987).

5. S. John, Phys. Rev. Lett 58, 2486 (1987).

6. V.S. Gorelik, Laser Physics 18, 1479 (2008).

7. V.S. Gorelik, P.P. Sverbil, V.V. Filatov, et al., Photonics Nanostructures: Fundam. Appl. 32, 6 (2018).

8. C. van de Hulst, Light scattering by small particles (N.Y.: Wiley), 470 (1957).

9. K.I. Zaytsev, S.O. Yurchenko, Appl. Phys. Lett. 105, 269 (2014).

10. S.O. Yurchenko, K.I. Zaytsev, E.A. Gorbunov, et al., J. Phys. D: Appl. Phys. 50, 055105 (2017).

11. V.S. Gorelik, Eur. Phys. J. Appl. Phys. 49, 33007 (2010). 